\title{
Retinal abnormatilites as a diagnostic or prognostic marker of schizophrenia
}

\author{
Ladislav Hosaka, Omar Sery b,c, Evgenii Sadykova, Jan Studnicka ${ }^{d}$
}

\begin{abstract}
The review is a summary of structural and functional changes in the human retina observed in patients with schizophrenia. The main focus is on the potential of these changes to serve as schizophrenia-specific biomarkers accessible to clinicians. We identified three features of the retina that can be detected non-invasively in humans and which appear to show charateristic changes in schizophrenia: (1) retinal microvasculature displaying abnormally wide venules; (2) electroretinograms indicating altered function of photoreceptors or other cells in the retinal component of the visual pathway; (3) optical coherence tomography pointing to structural differences between the retinae of patients with schizophrenia and those of healthy volunteers. We propose that the most feasible approach to evaluating the data would be to study the genetic and epigenetic background of the schizophrenia-associated retinal abnormalities and establish their significance and specificity as potential biomarkers for the disease. The studies should include longitudinal observations focusing on the possible involvement of medication and comorbid conditions in the mechanism of the disease; a comparison of schizophrenia with other mental disorders; and investigating retinal abnormalities in animal models of psychoses. Biomarkers identified in the process could represent an important addition to the arsenal of non-invasive techniques available to both clinicians and researchers. These novel biomarkers could facilitate research of the biological basis of psychosis and help to address the diagnostic, predicitive, preventative, prophylactic and therapeutic aspects of schizophrenia.
\end{abstract}

Key words: schizophrenia, retina, biomarker, endophenotype, diagnostics, therapy

Received: February 15, 2018; Accepted with revision: June 11, 2018; Available online: June 29, 2018 https://doi.org/10.5507/bp.2018.035

${ }^{a}$ Department of Psychiatry, Charles University, Faculty of Medicine in Hradec Kralove and University Hospital Hradec Kralove, Czech Republic 'Laboratory of Neurobiology and Molecular Psychiatry, Department of Biochemistry, Faculty of Sciences, Masaryk University, Brno, Czech Republic

'Institute of Animal Physiology and Genetics, Academy of Sciences of the Czech Republic, Brno, Czech Republic

${ }^{d}$ Department of Ophthalmology, Charles University, Faculty of Medicine in Hradec Kralove and University Hospital Hradec Kralove, Czech Republic

Corresponding author: Ladislav Hosak, e-mail: hosak@lfhk.cuni.cz

\section{INTRODUCTION}

Schizophrenia affects about $0.5-1 \%$ of the population. However, due to its chronic nature, it is among the ten leading causes of human disability. As such, schizophrenia has a major impact on social and health costs ${ }^{1}$. A patient's abilities to think clearly, to experience emotions, to respond adequately to commonly encountered life situations and to participate in normal social activities in a meaningful way are all severely compromised; this has a major impact on the affected individual's quality of life. Even though most of the patients with schizophrenia are, at least to a degree, responsive to pharmacological intervention, satisfactory recovery is only achieved in about a third of patients, with about $20-40 \%$ remaining resistant to treatment $^{2}$. Based on a systematic review of all original studies concerning suicide in schizophrenia published since 2004, Hor and Taylor concluded that the lifetime risk of suicide in patients with schizophrenia is approximately $5 \%\left(\right.$ ref. $\left.^{3}\right)$.

Specific biomarkers of schizophrenia have been sought for decades. Such markers would not only be of immense significance for clinicians as tools in diagnostics, prevention and for selecting individualized treatment for patients, but could also help to change the definition of schizophrenia, shifting it away from the present emphasis on the heterogeneity towards (a) more manageable and specific concept(s). Neurochemistry, neurophysiology, electrophysiology, brain imaging, cognitive functions, genetics, epigenetics, proteomics or metabolomics have all been studied in relation to potential biomarkers but, to date, there has been no satisfactory outcome and more efforts in this direction are needed ${ }^{47}$.

Investigating the living brain to better understand the biological underpinning of mental disorders remains one of the major challenges of psychiatry. Novel approaches are needed to study brain functions indirectly; observing the human retina could be one of them. The retina is an outgrowth of the diencephalon (i. e. related to the thalamus and hypothalamus), thus a part of the central nervous system (CNS). However, the only "true" neurons of the retina are the ganglion cells which form the sole retinal projection (via the optic nerve and optic tract) to the rest of the CNS. Amacrine cells express several neurotransmitters including dopamine (also expressed by the enigmatic "displaced" amacrine cells) (ref. ${ }^{8}$. The amacrine cell layer, with all its synaptic complexities, is sometimes likened to a "reticular formation" of the retina. The inner retina (ganglion and amacrine cell layers) receive their blood supply from branches of the central retinal artery while the outer retina (the recep- 
tors: rods and cones) receive no direct blood supply at all and rely entirely on the diffusion of oxygen from other retinal layers and across the choroid. Yet the receptor layer has probably the highest energy requirements of any tissue, possibly on account of its unusual ionic channel characteristics ${ }^{9}$. This critical balance of energy supply and energy requirements should make the retina highly vulnerable to small changes in energy metabolism, but it appears to be quite resilient. It is conceivable that any small genetically imposed metabolic or structural deficiencies would be readily compensated during early development. Such compensations could leave behind subtle yet detectable changes in the retinal anatomy or pathophysiology. Retinal changes thus observed in patients with a particular psychiatric disorder may reflect structural and functional disturbances which exist throughout the brain tissue and are specifically associated with the etiology of the disorder. The retina may be investigated by observing microvascular abnormalities, or by looking for functional and structural changes using electrophysiological recording (electroretinogram) or optical coherence tomography (OCT). Such ophthalmological examinations are accessible to clinicians and could be used when researching the pathogenesis of schizophrenia ${ }^{10,11}$.

The aim of the present review is to sum up the recent knowledge of retinal changes in schizophrenia in order to facilitate further research.

\section{VISUAL DEFICITS IN SCHIZOPHRENIA}

The study by Martinez et al. used functional magnetic resonance imaging to investigate the neurophysiological bases of visual processing deficits in schizophrenia, particularly the role of functional changes in the magnocellular cellular component of the visual pathway ${ }^{12}$. Subjects (schizophrenia $n=11$; schizoaffective disorder $n=2$; healthy volunteers $n=11$ ) were presented with continually changing images specifically designed to produce differential activation of magnocellular versus parvocellular systems based on their differential processing of contrast $^{12}$. Changes in the hemodynamics produced by the experimental visual input were mapped over the occipital (visual) cortex (particularly the visual areas V1 and V2) and the rest of the brain. Schizophrenia patients showed reduced responses to low, but not high, spatial frequencies in several regions of the occipital, parietal, and temporal lobes. According to the authors, these findings support the hypothesis that schizophrenia is associated with impaired functioning of the magnocellular stream of the visual pathway and suggest that these sensory processing deficits may contribute to higher-order cognitive deficits in working memory, executive functioning, and attention.

\section{MICROVASCULAR ABNORMALITIES}

Meier et al. tested the hypothesis that the retinal microvessels reflect familial vulnerability to psychotic symptoms ${ }^{13}$. Participants were 531 adolescent and young adult twins who took part in the Brisbane Longitudinal Twin Study and the Twins Eye Study in Tasmania. The twins had photographs taken of their retina when they were adolescents or young adults ( $\mathrm{M}$ age $=20.6$ years $)$, and the retinal vessel diameter was assessed using computer software. The twins completed an assessment of psychosis symptoms approximately six years later. The authors compared the retinal venular diameters of individuals with one or more symptoms of psychosis $(n=45)$ to their unaffected co-twins $(n=24)$ and to controls $(n=462)$. Individuals with one or more symptoms of psychosis had wider venules (standardized mean $=0.29$ ) than controls ( standardized mean $=-0.04 ; P=0.03$ ); and unaffected co-twins had venular diameters that were intermediate ( standardized mean $=0.13$ ) between the two groups. This finding suggests that wide venules may serve as a marker of vulnerability to psychosis symptoms. There were no differences in the arteriolar diameter between individuals with and without symptoms of psychosis.

We recently reviewed what is known of microvascular abnormalities in schizophrenia ${ }^{14}$. Based on seven published studies generally consistent with the hypothesis of microvascular pathology and brain inflammation as parts of the pathogenesis in schizophrenia, we suggested a specific approach to further research and for clinical exploitations of the anticipated findings. It can be summarized in the following five points: (1) to assess whether the microvascular abnormality as observed by retinal imaging fulfils the criteria for the schizophrenia endophenotype; (2) to carry out a detailed imaging examination of retinal anatomy, including that of the microvasculature, in individuals deemed at high-risk of developing schizophrenia; (3) to determine whether the brain functional magnetic resonance imaging (fMRI) findings and cognitive abilities of schizophrenia patients in both longitudinal and crosssectional studies are associated with the microvascular abnormalities identified by the retinal imaging; (4) to determine if there is a correlation between microvascular retinal pathology and the positive or negative schizophrenia symptoms (this point could include an investigation of whether childhood maltreatment results in, or is in any way associated with, abnormalities in retinal imaging); (5) to analyse the genetic background of schizophrenia retinal microvascular pathology and to apply anti-inflammatory agents in the treatment and prevention of schizophrenia if brain vasculitis is confirmed.

\section{ELECTRORETINOGRAM}

Warner et al. measured the electroretinogram (ERG) of 9 patients with schizophrenia and 9 age and sex matched control subjects ${ }^{15}$. Schizophrenic subjects had significantly reduced a-wave amplitudes on the ERG when compared with control subjects and the a-wave amplitude was independent of the dose of antipsychotic agents being taken. The a-wave of the ERG is thought to reflect activity of the photoreceptor cells. According to the authors, these findings support the hypothesis that patients with schizophrenia have abnormalities of photoreceptor func- 
tion, perhaps a result of reduced levels of n-3 essential fatty acids in the cell membrane.

The aim of the study by Balogh et al. was to investigate the earliest stages of visual information processing using electroretinography in patients with schizophrenia $(n=26)$ and bipolar disorder $(n=17)$ and to compare these values with those of healthy control volunteers $(n=20)\left(\right.$ ref. $\left.^{16}\right)$. In the acute stage of the illness, patients with schizophrenia exhibited decreased a-wave amplitude as compared with healthy controls ( 41.5 vs $59.7 ; P=0.0001$ ). In patients with bipolar disorder, electroretinography measures were intact (56.6). At the baseline assessment, there was a significant negative relationship between a-wave amplitude and positive symptoms in schizophrenia $(\mathrm{r}=-0.51 ; P<0.05)$. After an 8-week follow-up period, clinical symptoms showed significant improvement and the amplitude of the a-wave significantly increased in patients with schizophrenia $(P=0.0002)$. At the follow-up assessment, there was no significant difference between patients with schizophrenia (52.0) and controls (59.9). According to the authors, the results indicate that retinal dysfunctions are specific for schizophrenia, as compared to bipolar disorder, and are confined to the acute stage of the illness.

Hebert et al. reported a specific electroretinographic anomaly in young, non-affected and non-medicated offspring at high genetic risk (HR) of schizophrenia (SZ) or bipolar disorder (BD) (ref. $\left.{ }^{17}\right)$. Electroretinography was performed in $29 \mathrm{HR}$ offspring having one parent affected by schizophrenia or bipolar disorder (mean age: 20.8 years) and 29 healthy control subjects (mean age: 20.6 years). The HRs' parents descended from multigenerational families affected by SZ or BD. Rod ERG (b-wave amplitude at $\mathrm{V}(\max ))$ in HRs was significantly lower than in control subjects $(P<0.0001$; effect size of -1.47$)$, whereas the cone ERG V(max) showed no difference $(P=0.27)$. No effects of gender, age, and seasons of testing were noted. The anomaly in retinal response $(\operatorname{rod} \mathrm{V}(\max )$ b-wave amplitude) was observed independently of the parents' diagnosis (SZ; $P=0.007$, effect size of -1.09 ; BD: $P<0.0001$, effect size of -1.88$)$ and was present in both the younger and older HRs (effect size of -1.6 and -1.8 , respectively). According to the authors, a rod retinal response anomaly before the age of the disease incidence may represent an early and specific biomarker of risk and prove meaningful for further genetic and prevention research.

The aim of the study by Hebert et al. was to clarify and enhance electroretinogram results in schizophrenia and confirm whether adult patients would exhibit the ERG anomalies that the authors of the study had previously observed in children/adolescents at genetic risk ${ }^{17,18}$. The present sample consisted of 105 schizophrenia patients (mean age 40.1, 79\% males, pupil size 3.9) and 150 controls (mean age 40.6, 62\% males, pupil size 3.8 ). Only patients who were not refractory to treatment were included. An electroretinogram was recorded at various intensities in the non-dilated pupil. Results showed that patients differed from controls on cone a-wave amplitude, mixed rod-cone b-wave amplitude and pure rods b-wave amplitude, with significant effect sizes $(\sim 0.6)$ and a trend for reduced mixed rod-cone a-wave amplitude. Novel anoma- lies with significant large effect sizes were also found; a cone b-wave implicit time and cone b-wave amplitude anomalies. No correlation between the electroretinogram and duration of illness, antipsychotic doses or tobacco use was found. The authors confirmed that the rod bwave and cone a-wave amplitude reductions identified in healthy children/adolescents at risk were present in adult patients ${ }^{17,18}$. According to Hebert et al., the latter electroretinogram anomalies would be present many years before the disease onset, making them valuable research tools to address the pathophysiology and neurodevelopmental course of schizophrenia ${ }^{18}$.

\section{OPTICAL COHERENCE TOMOGRAPHY}

The study by Chu et al. explored the potential of the retinal nerve fibre layer (RNFL) and macular volume (MV) to detect axonal abnormalities in vivo in schizophrenia and to show their correlations with clinical features ${ }^{19}$. Optical coherence tomography was performed in 49 patients (38 schizophrenia, 11 schizoaffective disorder) and 40 healthy controls matched for age and gender. Group comparisons were made between the whole retina, the quadrant RNFL thickness and the MV using multi-level analyses. Associations were sought between RNFL and MV in terms of symptom severity (positive/ negative). Patients and controls had similar whole retina RNFL thickness $(P=0.86)$ and MV $(P=0.64)$, but RNFL in the right nasal quadrant of the schizoaffective group was thinner than in the schizophrenia group $(P=0.02)$. Positive symptom severity was associated with smaller MV (right $\beta=-0.54, P=0.02$; left $\beta=-0.49, P=0.04$ ). According to the authors, normal MV and RNFL thickness suggests that unmyelinated axons in patients with schizophrenia/schizoaffective disorder remain unaffected. Chu et al. suggested that longitudinal studies using higher resolution OCT would clarify whether progressive RNFL and MV changes occur and whether they can be used as state or trait markers in schizophrenia ${ }^{19}$.

Using spectral-domain optical coherence tomography (SD-OCT), Lee et al. determined structural retinal nerve fiber layer changes in schizophrenia patients and investigated whether the structural changes were related to the duration of the illness ${ }^{20}$. The authors recruited a total of 30 schizophrenic patients and 30 age-matched controls for the study. The schizophrenic patients were further subdivided into acute $(n=5)$, chronic $(n=13)$, and long-term chronic $(n=12)$ subgroups depending on the duration of their illness. Using SD-OCT, the peripapillary RNFL thickness, macula thickness, and macula volume measurements of schizophrenic patients and the control subjects were measured and compared at each location. Schizophrenic patients showed a statistically significant reduction in overall peripapillary RNFL thickness (cases, $94.70 \mu \mathrm{m}$; controls, $103.53 \mu \mathrm{m} ; P<0.001$ ), macula thickness (cases, $269.26 \mu \mathrm{m}$; controls, 284.83 $\mu \mathrm{m} ; P<0.001$ ), and macula volume (cases, $9.61 \mathrm{~mm}^{3}$; controls, $\left.10.17 \mathrm{~mm}^{3} ; P<0.001\right)$. Chronic and long-term chronic schizophrenic patients were found to have sig- 
nificant peripapillary RNFL thinning, macula thinning, and reduction of macula volume when compared to controls $(P<0.001)$. There also was a statistically significant reverse correlation $(P<0.05)$ of peripapillary RNFL thickness $(\mathrm{r}=-0.36)$, macula thickness $(\mathrm{r}=-0.38)$, and macula volume reduction $(\mathrm{r}=-0.36)$ with the duration of the schizophrenic illness. These results indicate that RNFL and macula thickness, as well as macula volume measurements are reduced in schizophrenic patients. The degree of thinning and reduction was more significant in the chronic phase of the disease and correlated with the duration of illness. According to the authors, these findings demonstrate that SD-OCT can be a useful tool for monitoring the progression of schizophrenia.

Ascaso et al. studied retinal optical coherence tomography measures of patients with schizophrenia with respect to healthy controls and evaluated possible differences between recent illness episode (RIE) and non-recent illness episode (NRIE) patients ${ }^{21}$. Thirty schizophrenia patients were classified as RIE $(n=10)$ or NRIE $(n=20)$ and compared with 30 matched controls. The patients had a significantly thinner retinal nerve fiber layer in overall measurements $(P=0.006)$, and in the nasal, superior and inferior quadrants $(P=0.018-0.047)$. Macular inner ring thickness $(P=0.004)$ and macular volume $(P=0.04)$ were also significantly smaller in patients than in controls. Compared with controls, only NRIE (but not RIE) patients had significantly reduced RNFL overall measures, superior RNFL, nasal RNFL, macular volume, and macular inner ring thickness $(P=0.004-0.036)$. The authors concluded that retinal parameters observed using OCT in schizophrenia patients could be studied as potential state biomarkers of the disorder.

Yilmaz et al. investigated the retina and macular thickness in patients with schizophrenia and compared the results with those of healthy controls ${ }^{22}$. Sixty-eight eyes of 34 patients with schizophrenia and 60 eyes of 30 randomly selected healthy volunteers were involved in the study. Measurements of peripapillary retinal nerve fiber layer thickness, macula thickness, and macula volume were taken in both eyes using spectral-domain optical coherence tomography. The average and nasal RNFL thicknesses were found to be significantly lower in the patient group than the control group $(P=0.030, P=0.04$, respectively). Macular thicknesses of nasal outer and inferior outer quadrants in the patient group were significantly lower than in the control group $(P=0.009, P=0.027$, respectively). The average macular thickness as well as the macular thicknesses in the superior outer, superior inner, temporal outer, temporal inner, nasal inner, and inferior inner areas were non-significantly lower in the patient group compared to the control group.

Celik et al. measured the RNFL thickness and the ganglion cell layer (GCL) and internal plexiform layer (IPL) volumes in 40 treatment refractory patients with schizophrenia, 41 treatment responsive schizophrenia patients and 41 controls using spectral-OCT; correlations between the disease severity and OCT measurements were also evaluated ${ }^{23}$. The global RNFL thickness and GCL and IPL volumes were decreased in patients with schizophrenia compared to the controls. In addition, the GCL and IPL volumes were lower in the treatment refractory patients with schizophrenia compared to the treatment responsive patients. Using parameters such as the Positive and Negative Syndrome Scale and Clinical Global Impression scores, disease duration and number of hospitalizations, correlations between the GCL and IPL volumes and disease severity were stronger than the correlations between the RNFL and the disease parameters. According to Celik et al., the findings suggest that OCT can be used to detect neurodegeneration in schizophrenia and that GCL and IPL volumes can also be used to monitor the progression of neurodegeneration ${ }^{23}$.

\section{DISCUSSION}

The current literature suggests that schizophrenia may be associated with wider venules in the retina, abnormalities of photoreceptor function and structural retinal changes. Some of these findings are also promising as a new schizophrenia endophenotype or vulnerability marker.

\section{Advantages of the research into the retina and schizophrenia}

As compared to other methods of in vivo CNS investigation, examination of the retina is generally easy-to-use, non-invasive, pain-free, quick and cheap. Because the retinal nerve fibre layer is unmyelinated and comprises the first axons of the visual pathway, it can be considered a unique anatomical model. As such, it may provide insight into the pathophysiological processes of diseases with a neurodegenerative character, like schizophrenia. From this point of view, the retina may be considered a "window to the brain" (ref. ${ }^{24}$ ).

It is necessary to state that schizophrenia is more considered to be a neurodevelopmental disorder, and neurodegeneration may only be seen in a part of the patients. The idea that schizophrenia has its origins in early development dates back at least to the modern classification of the syndrome by Emil Kraepelin and Eugen Bleuler. The neurodevelopmental hypothesis of schizophrenia is supported e.g. by the impact of abnormal gestational experience, association of schizophrenia with maturational deficits and recent knowledge on genetic and molecular mechanisms of schizophrenia. Because the evidence for neurodegeneration in schizophrenia is limited, neurodegenerative processes may only be relevant for a subgroup of patients ${ }^{2}$.

\section{Specificity of retinal abnormalities for schizophrenia}

Retinal abnormalities are not fully specific for schizophrenia. They can also be found in other neurodevelopmental neuropsychiatric disorders like autism. Song et al. discovered that the functional field of view is narrower in individuals with autismus spectrum disorder as compared to a group without autism due to a greater eccentricity from the fovea ${ }^{25}$. Ritvo et al. recorded electroretinograms for 27 autistic patients and 20 age- and sex-mateched healthy volunteers. Thirteen (48\%) of the autistic patients 
demonstrated subnormal b-wave amplitudes which may indicate abnormal retinal function ${ }^{26}$. A reduction of the b-wave in autism was confirmed by Constable et al. ${ }^{27}$. A reduction in retinal nerve fiber layer thickness in young adults with autism spectrum disorders was detected by Gialloreti et al. ${ }^{28}$. New knowledge on retinal abnormalities in autism can be expected within a few years.

\section{Suggestions of new research avenues}

The biological underpinning of anomalies in the schizophrenia electroretinogram remains unknown. Several genetic variants associated with enhanced risk for SZ and/ or BD can activate glycogen synthase kinase- 3 isozymes (GSK $3 \alpha$ and $\beta$ ). Lavoie et al. examined the potential contribution of GSK $3 \alpha$ and $\beta$ in the modulation of ERG in mice $^{29}$. GSK3 expression modulated some ERG parameters. The phenotype observed in prpGSK $3 \beta$ mice was consistent with observations made in human individuals with a high genetic risk for schizophrenia. ERG anomalies observed in GSK $3 \beta(+/-)$ and GSK $3 \alpha-\mathrm{KO}$ mice confirm an association between the rod and cone b-wave amplitude and the expression of GSK3 isozymes. According to Lavoie et al., changes in GSK3 expression or activity may explain some ERG anomalies in HRs and schizophrenia patients ${ }^{29}$. This calls for further research into the genetic and epigenetic background of retinal abnormalities in schizophrenia.

Possible associations of retinal markers with individual dimensions of schizophrenia (positive, negative and cognitive symptoms) including symptom severity should be sought for. The possible influence of gender should not be neglected.

Not only cross-sectional but also longitudinal studies in schizophrenia should be performed in order to detect the possible influence of the stage of the illness, antipsychotic treatment, and comorbid conditions on the retinal findings. It should be determined whether retinal abnormalities are a state marker or a trait marker of schizophrenia. Retinal differences between treatmentresponsive vs treatment-resistant schizophrenia patients may be revealed.

To assess the possible predictive value of retinal findings, non-medicated offspring at high genetic risk of schizophrenia should be examined repeatedly during their lives, whether or not schizophrenia occurs in them.

To establish whether retinal abnormalities in schizophrenia represent an endophenotype, healthy first-degree relatives of schizophrenia patients should be examined in additon to the patients and unrelated healthy volunteers.

Individual mental disorders (for example schizophrenia, schizoaffective disorder, delusional disorder, mood disorders with psychotic features, methamphetamineinduced psychosis, Alzheimer's disease and other dementias) should be compared to each other in terms of possible abnormal retinal findings in order to assess whether these ophthalmological anomalies are diseasespecific, or rather psychosis- or neurodegeneration-related.

Another promising possibility is to examine whether retinal abnormalities are associated with the clinical picture of experimental psychoses, for example induced by ketamine.
Schwitzer et al. suggest that the pattern electroretinogram (PERG), a retinal functional recording of the ganglion cells, might provide a relevant complementary measurement to enhance understanding of biological mechanisms in schizophrenia ${ }^{30}$. According to the authors, PERG protocols are good candidates to investigate dopamine transmission, a neurotransmitter known to be involved in schizophrenia. PERG is entirely measured in light conditions without any adaptation period. The PERG can be coupled with other retinal measurements, such as the flash electroretinogram (fERG), to provide a more thorough picture of the pathophysiology of schizophrenia. Adding functional measurements like PERG to imaging techniques such as OCT may provide a potential structure-function correlation. According to Schwitzer et al., PERG measurements have not yet been evaluated in schizophrenia patients ${ }^{30}$.

\section{Limitations of the research into the retina and schizo- phrenia}

The results of retinal examinations in schizophrenia may be biased by the effect of the illness, chronic drug therapy or comorbid conditions. Nevertheless, the same holds true for other investigation methods like brain imaging or electrophysiology. Another limitation is that examination of retina only covers a small part of the central neuronal tissue, whilst the parts of the brain which are larger and more important in schizophenia (mesolimbic system, prefrontal cortex) are not depicted.

\section{CONCLUSION}

Morphological and functional examination of the retina in schizophrenia appears to be a suitable accessory to other research methods like brain imaging, electrophysiology or neuropsychology. It will help us enhance our understanding of the neurobiological mechanisms in schizophrenia and their possible associatons with clinical symptoms. Revealed retinal anomalies may become diagnostic, predictive, therapeutic, preventative or prophylactic biomarkers in schizophrenia. This will contribute to better diagnostics and improved individualized treatment of this serious mental disorder and, last but not least, reduce its stigma.

\section{Search strategy and selection criteria}

Our research strategy was aimed at evaluating studies on retinal changes in schizophrenia against healthy volunteers or other major mental disorders. Scientific articles from 1990 to 2017 were searched using the PubMed database. The key words used included "retina" and "schizophrenia”. Only English language papers were reviewed.

\section{ABBREVIATION}

BD, Bipolar disorder; CNS, Central nervous system; ERG, Electroretinogram; fERG, Flash electroretinogram; fMRI, Functional magnetic resonance imaging; 
GCL, Ganglion cell layer; GSK3, Glycogen synthase kinase-3 isozymes; HR, High genetic risk; IPL, Internal plexiform layer; MV, Macular volume; NRIE, Non-recent illness episode; OCT, Optical coherence tomography; PERG, Pattern electroretinogram; RIE, Recent illness episode; RNFL, Retinal nerve fibre layer; SD-OCT, Spectral-domain optical coherence tomography; SZ, Schizophrenia.

Acknowledgement: Our work was supported by the Research Grant of the Agency for Medical Research, Ministry of Health of the Czech Republic 16-27243A.

Author contributions: LH: literature search and manuscript writing; OŠ, ES, JS: literature search and critical reading; all authors: manuscript revision.

Conflict of interest statement: The authors declare there are no conflicts of interest regarding the publication of this article.

\section{REFERENCES}

1. Murray CJL. The global burden of disease: a comprehensive assess ment of mortality and disability from diseases, injuries, and risk factors in 1990 and projected to 2020. Cambridge: Harvard School of Public Health on behalf of the World Health Organization and the World Bank; 1996.

2. Weinberger DR, Harrison PJ. Schizophrenia. Chichester: WileyBlackwell; 2011.

3. Hor K, Taylor M. Suicide and schizophrenia: a systematic review of rates and risk factors. J Psychopharmacol 2010;24(4 Suppl):81-90.

4. Thibaut F, Boutros NN, Jarema M, Oranje B, Hasan A, Daskalakis ZJ, Wichniak A, Schmitt A, Riederer P, Falkai P, WFSBP Task Force on Biological Markers. Consensus paper of the WFSBP Task Force on Biological Markers: Criteria for biomarkers and endophenotypes of schizophrenia part I: Neurophysiology. World J Biol Psychiatry 2015; 16(5):280-90

5. Landek-Salgado MA, Faust TE, Sawa A. Molecular substrates of schizophrenia: homeostatic signaling to connectivity. Mol Psychiatry 2016;21(1):10-28.

6. Schmitt A, Rujescu D, Gawlik M, Hasan A, Hashimoto K, Iceta S, Jarema M, Kambeitz J, Kasper S, Keeser D, Kornhuber J, Koutsouleris N, Lanzenberger N, Maichow B, Saoud M, Spies M, Stöber G, Thibaut F, Riederer P, Falkai P, WFSBP Task Force on Biological Markers. Consensus paper of the WFSBP Task Force on Biological Markers: Criteria for biomarkers and endophenotypes of schizophrenia part II: Cognition, neuroimaging and genetics. World J Biol Psychiatry 2016;17(6):406-28.

7. Šerý O, Povová J, Balcar VJ. Perspectives in genetic prediction of Alzheimer's disease. Neuro Endocrinol Lett 2014;35(5):359-66.

8. Masland RH. The tasks of amacrine cells. Vis Neurosci 2012;29(1):3-9.

9. Nema HV. Textbook of Ophthalmology. New Delhi: Jaypee Brothers Medical Publishers; 2011.

10. Gracitelli CP, Abe RY, Diniz-Filho A, Vaz-de-Lima FB, Paranhos A Jr, Medeiros FA. Ophthalmology issues in schizophrenia. Curr Psychiatry Rep 2015;17(5):28.

11. Silverstein SM, Rosen R. Schizophrenia and the eye. Schizophr Res Cogn 2015;2(2):46-55.

12. Martinez A, Hillyard SA, Dias EC, Hagler DJ Jr, Butler PD, Guilfoyle DN, Jalbrzikowski M, Silipo G, Javitt DC. Magnocellular pathway impairment in schizophrenia: evidence from functional magnetic resonance imaging. J Neurosci 2008;28(30):7492-500.
13. Meier MH, Gillespie NA, Hansell NK, Hewitt AW, Hickie IB, Lu Y, McGrath J, MacGregor S, Medland SE, Sun C, Wong TY, Wright MJ, Zhu G, Martin NG, Mackey DA. Retinal microvessels reflect familial vulnerability to psychotic symptoms: A comparison of twins discordant for psychotic symptoms and controls. Schizophr Res 2015;164(1-3):47-52.

14. Hosak L, Hakeem K, Raad M, Studnicka J. Is microvascular abnormality a new endophenotype in schizophrenia? Psychiatr Danub 2015;27(3):225-9.

15. Warner R, Laugharne J, Peet M, Brown L, Rogers N. Retinal function as a marker for cell membrane omega-3 fatty acid depletion in schizophrenia: a pilot study. Biol Psychiatry 1999;45(9):1138-42.

16. Balogh Z, Benedek G, Keri S. Retinal dysfunctions in schizophrenia. Prog Neuropsychopharmacol Biol Psychiatry 2008;32(1):297-300.

17. Hebert M, Gagne AM, Paradis ME, Jomphe V, Roy MA, Merette C, Maziade $M$. Retinal response to light in young nonaffected offspring at high genetic risk of neuropsychiatric brain disorders. Biol Psychiatry 2010;67(3):270-4.

18. Hebert M, Merette C, Paccalet T, Emond C, Gagne AM, Sasseville A, Maziade M. Light evoked potentials measured by electroretinogram may tap into the neurodevelopmental roots of schizophrenia. Schizophr Res 2015;162(1-3):294-5.

19. Chu EM, Kolappan M, Barnes TR, Joyce EM, Ron MA. A window into the brain: an in vivo study of the retina in schizophrenia using optical coherence tomography. Psychiatry Res 2012;203(1):89-94.

20. Lee WW, Tajunisah I, Sharmilla K, Peyman M, Subrayan V. Retinal nerve fiber layer structure abnormalities in schizophrenia and its relationship to disease state: evidence from optical coherence tomography. Invest Ophthalmol Vis Sci 2013;54(12):7785-92.

21. Ascaso FJ, Rodriguez-Jimenez R, Cabezon L, Lopez-Anton R, Santabarbara J, De la Camara C, Modrego PJ, Quintanilla MA, Bagney A, Gutierrez L, Cruz N, Cristobal JA, Lobo A. Retinal nerve fiber layer and macular thickness in patients with schizophrenia: Influence of recent illness episodes. Psychiatry Res 2015;229(1-2):230-6.

22. Yilmaz U, Kücük E, Ülgen A, Özköse A, Demircan S, Ulusoy DM, Zararsiz G. Retinal nerve fiber layer and macular thickness measurement in patients with schizophrenia. Eur J Ophthalmol 2016;26(4):375-8.

23. Celik M, Kalenderoglu A, Sevqi Karadag A, Bekir Egilmez O, Han-Almis $B$, Simsek A. Decreases in ganglion cell layer and inner plexiform layer volumes correlate better with disease severity in schizophrenia patients than retinal nerve fiber layer thickness: Findings from spectral optic coherence tomography. Eur Psychiatry 2016;32:9-15.

24. Schönfeldt-Lecuona C, Kregel T, Schmidt A, Pinkhardt EH, Lauda F, Kassubek J, Connemann BJ, Freudenmann RW, Gahr M. From Imaging the Brain to Imaging the Retina: Optical Coherence Tomography (OCT) in Schizophrenia. Schizophr Bull 2016;42(1):9-14.

25. Song Y, Hakoda Y, Sanefuji W, Cheng C. Can They See It? The Functional Field of View Is Narrower in Individuals with Autism Spectrum Disorder. PLoS One 2015;10(7):e0133237.

26. Ritvo ER, Creel D, Realmuto G, Crandall AS, Freeman BJ, Bateman JB, Barr R, Pingree C, Coleman M, Purple R. Electroretinograms in autism: a pilot study of b-wave amplitudes. Am J Psychiatry 1988;145(2):22932.

27. Constable PA, Gaigg SB, Bowler DM, Jägle H, Thompson DA. Full-field electroretinogram in autism spectrum disorder. Doc Ophthalmol 2016;132(2):83-99.

28. Emberti Gialloreti L, Pardini M, Benassi F, Marciano S, Amore M, Mutolo MG, Porfirio MC, Curatolo P. Reduction in retinal nerve fiber layer thickness in young adults with autism spectrum disorders. J Autism Dev Disord 2014;44(4):873-82.

29. Lavoie J, Hebert M, Beaulieu JM. Glycogen synthase kinase-3 overexpression replicates electroretinogram anomalies of offspring at high genetic risk for schizophrenia and bipolar disorder. Biol Psychiatry 2014;76(2):93-100.

30. Schwitzer T, Schwan R, Bernardin F, Jeantet C, Angioi-Duprez K, Laprevote V. Commentary: Anatomical constitution of sense organs as a marker of mental disorders. Front Behav Neurosci 2016;10:56. 